\title{
Development and Deployment of 'Smart Diagnostics': Next Generation Point of Care Sensors with Capacity to Learn
}

\author{
John T. McDevitt \\ Division of Biomaterials, New York University Dentistry, Tandon School of Engineering, \\ New York, NY
}

\begin{abstract}
While COVID-19 has yielded devastating consequences over the past few years, the global pandemic also has opened the door for acceleration of development of core diagnostic capabilities that have the potential to lead to lasting impact for our society. With this vantage point in mind, in the recent past the McDevitt laboratory has launched a series of efforts that target the development and deployment of 'smart diagnostics' that serve as distributed point of care sensor nodes with capacity to learn. These mini-sensor ensembles with embedded artificial intelligence integrate programmable chip-based diagnostic systems capable of multiplexed measurements alongside clinical decision support tools that utilize strategically chosen nonclinical data elements that elicit signatures that can be used to capture diseases before they spiral out of control. As such, these efforts link for the first-time the following five key disciplines: i) lab-on-a-chip technologies, ii) in vitro diagnostics, iii) -omics research, iv) artificial intelligence, and v) digital healthcare delivery systems.

Importantly, the combination of point-of-care medical microdevices and machine learning has the potential transform the practice of medicine. In this area, scalable lab-on-a-chip devices have many advantages over standard laboratory methods including portability, faster analysis, reduced cost, lower power consumption, and higher levels of integration and automation. Despite significant advances in medical microdevice technologies over the years, several remaining obstacles are preventing clinical implementation and market penetration of these novel medical microdevices. Similarly, while machine learning has seen explosive growth in recent years and promises to shift the practice of medicine toward dataintensive and evidence-based decision making, its uptake has been hindered due to the lack of integration between clinical measurements and disease determinations.

In this talk, our recent advances in 'smart diagnostics' will be highlighted. These smart diagnostics include single-use microfluidic cartridges that serve as fully integrated, self-contained devices that contain aqueous buffers suitable for automated completion of all assay steps within nontraditional healthcare settings. Further, a portable analyzer instrument is fashioned to integrate fluid delivery, optical detection, image analysis, and user interface, representing a universal system for acquiring, processing, and managing clinical data while overcoming many of the challenges facing the widespread clinical adoption of lab-on-a-chip technologies. Intimate linkages between these medical microdevices and cloud connected databases allows for early disease detection algorithms to be used to impact clinical.
\end{abstract}

This talk will summarize the development and deployment for 'smart diagnostics' for the areas for the following areas:

1) Oral cancer lesion adjunct testing: multiparameter single cell lesion diagnostics for precision medicine.

2) Cardiac scorecard: a clinical decision support system for a spectrum of cardiovascular diseases.

3) COVID-19 severity and immunity diagnostics: point of care tools to help better manage the corona virus. 